\title{
Numerical Absorbing Boundary Conditions for the Wave Equation
}

\author{
By Robert L. Higdon
}

\begin{abstract}
We develop a theory of difference approximations to absorbing boundary conditions for the scalar wave equation in several space dimensions. This generalizes the work of the author described in [8].

The theory is based on a representation of analytical absorbing boundary conditions proven in [8]. These conditions are defined by compositions of first-order, one-dimensional differential operators. Here the operators are discretized individually, and their composition is used as a discretization of the boundary condition. The analysis of stability and reflection properties reduces to separate studies of the individual factors. A representation of the discrete boundary conditions makes it possible to perform the analysis geometrically, with little explicit calculation.
\end{abstract}

1. Introduction. Consider the wave equation

$$
u_{t t}=c^{2}\left(u_{x x}+\sum_{j=1}^{k} u_{y_{j} y_{j}}\right)
$$

for $t>0$ on the spatial domain $\Omega=\left\{(x, y): x>0, y \in R^{k}\right\}$. Our goal is to find boundary conditions that cause wave motions from the interior of $\Omega$ to pass through the boundary $x=0$ without being reflected. Boundary conditions of this type are desirable in a number of physical problems. See, e.g., [2], [3], [4]. In general, it is not possible to find practical boundary conditions that do the above task perfectly.

In this paper we discuss difference approximations to absorbing boundary conditions of the form

$$
\left[\prod_{j=1}^{p}\left(\left(\cos \alpha_{j}\right) \frac{\partial}{\partial t}-c \frac{\partial}{\partial x}\right)\right] u=0,
$$

where $\left|\alpha_{j}\right|<\pi / 2$ for all $j$. An analysis of the analytical conditions (1.2) was given in [8]. Here we summarize some properties of (1.2).

(1) The condition (1.2) is satisfied exactly by any linear combination of plane waves traveling out of $\Omega$ at angles of incidence $\pm \alpha_{1}, \ldots, \pm \alpha_{p}$ with speed $c$. (In two space dimensions, consider $f\left(x \cos \alpha_{j}+y \sin \alpha_{j}+c t\right)$.) For such a linear combina-

Received February 3, 1986; revised July 9, 1986.

1980 Mathematics Subject Classification (1985 Revision). Primary 35L05, 65M99, 65N99.

Key words and phrases. Absorbing boundary conditions, wave equation, initial-boundary value problems. 
tion there is no reflection. In general, the reflection coefficient is

$$
-\prod_{j=1}^{p}\left(\frac{\cos \alpha_{j}-\cos \theta}{\cos \alpha_{j}+\cos \theta}\right)
$$

for sinusoidal plane waves traveling at angle of incidence $\theta$. Each factor in (1.3) has absolute value less than 1 when $|\boldsymbol{\theta}|<\pi / 2$.

(2) For any given $p$ and any given problem, the angles $\alpha_{j}$ can be chosen so as to distribute the zeros of (1.3) and thereby optimize the absorption properties of (1.2). In general, the choice of optimal $\alpha_{j}$ depends on the configuration of the problem. For example, the angles may be chosen to take advantage of a priori information about the directions from which particular wave motions approach the boundary. In practical situations one may need to limit the value of $p$ that is used; see the end of Section 3.

(3) In Proposition 9.1 of [8] it is shown that (1.2) provides a general representation of absorbing boundary conditions, in the following sense. If an absorbing boundary condition is based on a symmetric rational approximation to the portion of the dispersion relation corresponding to outgoing waves, then it is either

(a) equivalent to (1.2), for suitable $\alpha_{j}$ satisfying $\left|\alpha_{j}\right|<\pi / 2$; or

(b) unstable; or

(c) not optimal, in the sense that the coefficients in the boundary condition can be modified so as to reduce the magnitude of the reflection coefficient for all Fourier modes corresponding to nontangential incidence.

For example, the boundary conditions of Engquist and Majda [3], [4] are based on Padé approximations and are equivalent to (1.2) for $\alpha_{1}=\cdots=\alpha_{p}=0$.

Various rational approximations have also been used to construct one-way wave equations; see, e.g., [1], [6], [11], [12]. In certain physical problems such equations are applied throughout the spatial domain in order to model wave propagation in one direction but exclude propagation in the opposite direction. These equations can also be used as absorbing boundary conditions when (1.1) is used as the interior equation; in this case the factorization in remark (3) can be considered.

The radiating boundary conditions of Bayliss and Turkel [2] do not fit into the format described above. However, they resemble (1.2), with $\alpha_{j}=0$ and certain variable lower-order terms added to the various factors. The absorbing boundary conditions of Lindman [9] resemble the first-order version of (1.2), but include certain correction terms that involve values of several functions that need to be updated at each time step.

The purpose of the present work is to give a general analysis of difference approximations to (1.2). We discretize each factor with a first-order difference operator having a one-dimensional stencil. (See Section 3 for precise formulas.) The composition of these operators then gives an approximation to (1.2). This composition has the following properties.

(1) The stencil is one-dimensional, which simplifies implementation near corners of rectangular spatial domains.

(2) The analysis of stability and reflection properties reduces to separate studies of the individual factors. (See Sections 2 and 3.) This leads to major simplifications in the analysis. 
These remarks generally do not apply when one-way wave equations of order two or more are used as absorbing boundary conditions but are not factored as described above. (See, e.g., Section 8 of [8].)

The analysis in the present paper is performed using geometrical constructions in the complex plane and involves little explicit algebraic calculation. This analysis is based on the representation of discrete boundary conditions given by the operator $M\left(K, Z^{-1}\right)$ developed in Section 5. The representation may have application beyond the present problem; see the beginning of Section 6.

In [8] we analyzed and tested two types of discrete boundary conditions that are equivalent to certain discretizations of (1.2) for $\cos \alpha_{j}=\lambda=c \Delta t / \Delta x$. One of these methods, "space-time extrapolation", is extremely easy to implement. The purpose of the present work is to remove the restriction $\cos \alpha_{j}=\lambda$ and give a theory that covers a broader class of difference approximations to (1.2).

The outline of this paper is as follows. In Section 2 we make some preliminary comments. In Section 3 we describe the discrete boundary conditions used here and state the main results of the paper. These results are proved in Sections 4 through 7. Some numerical tests of the boundary conditions are given in Section 8 .

2. General Theoretical Framework. In this section we define some notation and describe the theoretical framework to be used here.

For the sake of notational simplicity only, we orient the paper to the case of two space dimensions $(k=1)$; the space domain is then given by $x>0$ and $y \in R$. However, the results also apply to problems in higher dimensions. At appropriate places we indicate the notational changes needed to describe and analyze the more general case.

Let $u_{j, m}^{n}$ denote an approximation to $u(j \Delta x, m \Delta y, n \Delta t)$, and let $K$ and $Z$ denote the forward shift operators with respect to $x$ and $t$, respectively, defined by $K u_{j, m}^{n}=u_{j+1, m}^{n}$ and $Z u_{j, m}^{n}=u_{j, m}^{n+1}$. We will consider boundary conditions of the form

$$
B\left(K, Z^{-1}\right) u_{0, m}^{n+1}=0,
$$

where $B$ is a polynomial in two variables having a nonzero constant term. The boundary conditions used later do not involve shifts in the $y$-direction. In the interior of $\Omega$ we will use the standard second-order centered difference approximation to (1.1); for two space dimensions this is given by

$$
\begin{aligned}
& \frac{u_{j, m}^{n+1}-2 u_{j, m}^{n}+u_{j, m}^{n-1}}{(\Delta t)^{2}} \\
& =c^{2}\left[\frac{u_{j+1, m}^{n}-2 u_{j, m}^{n}+u_{j-1, m}^{n}}{(\Delta x)^{2}}+\frac{u_{j, m+1}^{n}-2 u_{j, m}^{n}+u_{j, m-1}^{n}}{(\Delta y)^{2}}\right] .
\end{aligned}
$$

Extensions to other interior schemes are indicated at the beginning of Section 6 and at the end of Section 7.

In later analyses of boundary conditions we will use a stability criterion that is an analogue of one used by Gustafsson, Kreiss, and Sundström [5] to prove stability results for first-order hyperbolic systems in one space dimension. The criterion can be described as follows. 
Consider solutions of (2.2) that have the form

$$
u_{j, m}^{n}=\kappa^{j}\left(e^{i \eta \Delta y}\right)^{m} z^{n},
$$

where $\kappa$ and $z$ are complex and $\eta$ is real. The quantities in (2.3) must satisfy the "dispersion relation"

$$
\begin{aligned}
z-2+z^{-1}= & \left(c \frac{\Delta t}{\Delta x}\right)^{2}\left(\kappa-2+\kappa^{-1}\right) \\
& +\left(c \frac{\Delta t}{\Delta y}\right)^{2}\left(e^{i \eta \Delta y}-2+e^{-i \eta \Delta y}\right) .
\end{aligned}
$$

In the case of higher-dimensional problems, the last term in (2.4) is replaced by a sum of such terms.

For each $z$ and each $\eta \Delta y$, there are two solutions $\kappa_{1}, \kappa_{2}$ of (2.4). In Section 4 we show that if $|z|>1$, then $\kappa_{1}$ and $\kappa_{2}$ can be defined so that $\left|\kappa_{1}\right|<1$ and $\left|\kappa_{2}\right|>1$. When $|z|=1$ we define $\kappa_{1}$ and $\kappa_{2}$ to be limits of values corresponding to $|z|>1$. The stability criterion is

$$
B\left(\kappa_{1}, z^{-1}\right) \neq 0 \text { whenever }|z| \geqslant 1 \text {, for all } \eta \Delta y .
$$

A physical interpretation of (2.5) is given in [8]. (Also see Trefethen [10].) A related, but different, interpretation is given below.

In later sections we will consider boundary conditions defined by operators of the form

$$
B\left(K, Z^{-1}\right)=\prod_{j=1}^{p} D_{j}\left(K, Z^{-1}\right),
$$

where each $D_{j}$ is a discretization of a factor in (1.2). The criterion (2.5) amounts to requiring $D_{j}\left(\kappa_{1}, z^{-1}\right) \neq 0$ for each $j$. The factors can thus be analyzed individually.

Reflection properties of boundary conditions will be described as follows; cf. [3], [8], [10]. Let

$$
e^{i \xi x+i \eta y+i \omega t}
$$

denote an oscillatory wave. This can be written in the form (2.3), with

$$
\kappa=e^{i \xi \Delta x}, \quad z=e^{i \omega \Delta t} .
$$

Consider a linear combination

$$
c_{1} \kappa_{1}^{j} e^{i \eta y_{z} z^{n}}+c_{2} \kappa_{2}^{j} e^{i \eta y_{z} z^{n}},
$$

where subscripts 1 and 2 correspond to group velocity pointing into and out of the spatial domain $\Omega$, respectively. This is consistent with the definition of $\kappa_{1}$ and $\kappa_{2}$ given in connection with (2.5); see Section 4. When (2.9) is inserted into the boundary condition (2.1), the result is the reflection coefficient

$$
R(\eta, z)=\frac{c_{1}}{c_{2}}=-\frac{B\left(\kappa_{2}, z^{-1}\right)}{B\left(\kappa_{1}, z^{-1}\right)} .
$$

(In general, one would consider wave packets formed by integrating with respect to $\eta$ and $\omega$, but the analysis reduces to separate studies of each pair $(\eta, \omega)$. Also see the discussion of transforms given below.) We want $|R(\eta, z)|$ to be as small as possible. 
If a boundary condition has the factored form (2.6), then (2.10) implies

$$
R(\eta, z)=-\prod_{j=1}^{p}\left(-R_{j}(\eta, z)\right),
$$

where $R_{j}$ is the reflection coefficient associated with $D_{j}$. The reflection properties can thus be studied one factor at a time.

The reflection coefficient leads to an interpretation of (2.5) that suggests the validity of this criterion. Suppose $|z|>1$. Then (2.3) consists of values of modes $\exp (\gamma x+i \eta y+s t)$ for $\operatorname{Re} s>0$, where $\kappa=\exp (\gamma \Delta x)$ and $z=\exp (s \Delta t)$. The general solution of the interior difference equation can be built up from these modes by means of Fourier transforms in $y$ and Laplace transforms in $t$. (The process is an analogue of one given in Section 5 of [7] for first-order hyperbolic systems. Reduce the transformed interior equation to a one-step system of difference equations in $x$, and analyze the solution.) The limit $|z| \rightarrow 1$ corresponds to $\Delta t \rightarrow 0$, if $\operatorname{Re} s$ is fixed.

Now consider (2.9), but with $|z|>1,\left|\kappa_{1}\right|<1$, and $\left|\kappa_{2}\right|>1$. The first and second terms can still be associated with incoming and outgoing waves, respectively. (See, e.g., [7], [10], or Section 4.) The stability criterion (2.5) then means that it is possible to solve for incoming modes in terms of outgoing modes (see (2.10)), and the solvability is uniform as the mesh is refined. This interpretation is an analogue of an interpretation given in [7] of the Kreiss well-posedness criterion for first-order hyperbolic systems.

3. Difference Approximations to (1.2); Statement of Results. In this section we define some discretizations of (1.2) and state the main results of the paper. Let

$$
(\cos \alpha) \frac{\partial}{\partial t}-c \frac{\partial}{\partial x}
$$

denote a typical factor in (1.2). This will be approximated by

$$
\begin{aligned}
D\left(K, Z^{-1}\right)= & (\cos \alpha)\left(\frac{I-Z^{-1}}{\Delta t}\right)[(1-a) I+a K] \\
& -c\left(\frac{K-I}{\Delta x}\right)\left[(1-b) I+b Z^{-1}\right] .
\end{aligned}
$$

The coefficients $a$ and $b$ give weighted space and time averages of the time and space differences, respectively. This formulation includes the forward Euler, backward Euler, and box scheme approximations to (3.1). (See Section 5.2.) At this point we place no restrictions on $a$ and $b$, except that they be real.

We will discretize (1.2) by a composition of the form

$$
\left(\prod_{j=1}^{p} D_{j}\left(K, Z^{-1}\right)\right) u_{0, m}^{n+1}=0 .
$$

The angle $\alpha$ and the coefficients $a$ and $b$ may depend on $j$.

According to remarks made in Section 2, the analysis of (3.3) reduces to a study of individual operators of the form (3.2). In order to perform this analysis, we first develop the representation of $D\left(K, Z^{-1}\right)$ given in Lemma 1. This representation is used to prove the reflection property in Theorem 1 and the stability result in Lemma 2. The conclusions of Lemma 2 are then translated into the statement about $D\left(K, Z^{-1}\right)$ given in Theorem 2. The proofs of these results are given in later sections. 
The lemmas and theorems are stated for the case of two space dimensions. The corresponding statements about higher-dimensional problems $\left(y \in R^{k}\right)$ are obtained by replacing $(c \Delta t / \Delta y)^{2}$ with $\sum\left(c \Delta t / \Delta y_{j}\right)^{2}$ and $\eta \Delta y$ with $\eta \cdot \Delta y$.

Lemma 1. $D\left(K, Z^{-1}\right)$ can be manipulated to yield

$$
\begin{aligned}
\frac{\Delta x}{c}[ & \left.a+b\left(\frac{\lambda}{\cos \alpha}\right)\right] D\left(K, Z^{-1}\right) \\
& =I-\left[I+r_{Z}\left(Z^{-1}-I\right)\right]\left[I+r_{K}(K-I)\right] \\
& =M\left(K, Z^{-1}\right),
\end{aligned}
$$

where $\lambda=c \Delta t / \Delta x$ and

$$
r_{Z}=a\left(\frac{\cos \alpha}{\lambda}\right)+b ; \quad r_{K}=\left(\frac{\lambda}{\cos \alpha}\right) r_{Z}
$$

Remarks. A motivation for the form of $M\left(K, Z^{-1}\right)$ is given in Subsection 5.1. There, we use properties of the dispersion relation (2.4) to develop a class of discrete boundary conditions that are approximately satisfied by outgoing waves but are not satisfied by incoming waves. This derivation is the process by which the author originally obtained $M\left(K, Z^{-1}\right)$. This development uses properties of the interior difference scheme and does not consider the possibility of consistency with analytical boundary conditions.

However, Lemma 1 shows that if $a+b(\lambda / \cos \alpha) \neq 0$, then the behavior of $M\left(K, Z^{-1}\right)$ is actually equivalent to that of $D\left(K, Z^{-1}\right)$. This fact will be the basis of essentially all of the later analysis, for the following reason.

The analysis of stability and reflection properties of $D\left(K, Z^{-1}\right)$ requires the analysis of the complex-valued functions $D\left(\kappa_{1}, z^{-1}\right)$ and $D\left(\kappa_{2}, z^{-1}\right)$. (See Theorems 1 and 2.) Here $|z| \geqslant 1,\left|\kappa_{1}\right| \leqslant 1,\left|\kappa_{2}\right| \geqslant 1$. If the given form of $D\left(K, Z^{-1}\right)$ were to be used, the analysis would appear to require a great deal of tedious algebraic manipulation. Such manipulation is a traditional feature of applications of the stability theory of Gustafsson, Kreiss, and Sundström [5]. However, the situation is different in the case of the equivalent operator $M\left(K, Z^{-1}\right)$. The function $1+$ $r_{Z}\left(z^{-1}-1\right)$ maps the domain $|z| \geqslant 1$ to a certain disk in the complex plane; a similar comment holds for $1+r_{K}(\kappa-1)$. In later sections it will be seen that much of the analysis of stability and reflection can then be performed by studying simple geometrical properties of certain mappings in the complex plane. This does a great deal to simplify the analysis.

The operator $M\left(K, Z^{-1}\right)$ does not help in the analysis of the special case $a+b(\lambda / \cos \alpha)=0$. However, this case will be handled easily by separate arguments.

If $a$ and $b$ are varied so that the quantities $r_{Z}$ and $r_{K}$ in (3.5) do not change, then $M\left(K, Z^{-1}\right)$ does not change. Thus different discretizations of $\partial / \partial t$ and $\partial / \partial x$ in (3.1) can lead to equivalent operators (3.2). In particular, no generality is lost by assuming $a=b$ in (3.2). This remark includes the case $a+b(\lambda / \cos \alpha)=0$; see (5.9).

In the remainder of this section we assume that the interior difference scheme satisfies the Courant-Friedrichs-Lewy condition

$$
\left(c \frac{\Delta t}{\Delta x}\right)^{2}+\left(c \frac{\Delta t}{\Delta y}\right)^{2} \leqslant 1 .
$$


THEOREM 1 (Reflection property). If $|z|=\left|\kappa_{1}\right|=\left|\kappa_{2}\right|=1$ and $\kappa_{1} \neq \kappa_{2}$, then

$$
\left|\frac{D\left(\kappa_{2}, z^{-1}\right)}{D\left(\kappa_{1}, z^{-1}\right)}\right|<1 \text {. }
$$

This holds for all choices of $a$ and $b$ and all $\eta \Delta y$.

Remarks. Theorem 1 implies that the reflection coefficient for the general boundary condition (3.3) has absolute value less than 1 for purely oscillatory modes, except when $\kappa_{1}=\kappa_{2}$ (cf. (2.11)). The constraint $\kappa_{1} \neq \kappa_{2}$ means, essentially, that the case of zero normal group velocity is excluded. For details, see the proof of Theorem 1 in Subsection 5.3. The exceptional case is of no consequence.

The above theorem applies to all oscillatory waves admitted by the interior difference scheme. It is not restricted just to those waves that are resolved well by the grid and thus described by consistency with (1.1) and (1.2).

Lemma 2 (Stability for $M\left(K, Z^{-1}\right)$ ). Suppose that $r_{Z}$ and $r_{K}$ are positive. If

$$
\left(2 r_{Z}-1\right)\left(2 r_{K}-1\right) \leqslant 1 \text {, }
$$

and if strict inequality holds either in (3.8) or in the CFL condition (3.6), then the operator $M\left(K, Z^{-1}\right)$ in (3.4), (3.5) satisfies the stability condition

$$
M\left(\kappa_{1}, z^{-1}\right) \neq 0, \quad \text { for }|z| \geqslant 1 \text { except if } z=\kappa_{1}=1 \text {, and for all } \eta \Delta y .
$$

If strict inequality holds in (3.6), then the constraint (3.8) can be relaxed slightly; necessary and sufficient conditions for (3.9) are given at the end of the proof of Lemma 2 in Section 6.

If $r_{Z}$ and $r_{K}$ are negative, then (3.9) is satisfied; no constraints need be placed on $r_{Z}$ and $r_{K}$ in this case.

Remarks. If $M\left(K, Z^{-1}\right)$ is used to represent $D\left(K, Z^{-1}\right)$, then $r_{Z} / r_{K}=(\cos \alpha) / \lambda$ $>0$. (See (3.5).) Thus it is no restriction to assume that $r_{Z}$ and $r_{K}$ have the same sign. Some numerical computations described in Section 8 suggest that, in practice, $r_{Z}$ and $r_{K}$ should be positive. The exceptional case $M(1,1)=0$ in (3.9) is discussed below.

Lemma 2 is used to help prove the following, final result.

THEOREM 2 (Stability for $\left.D\left(K, Z^{-1}\right)\right)$. If

$$
a\left(\frac{\cos \alpha}{\lambda}\right)+b \leqslant \frac{1}{2}\left(\frac{\cos \alpha}{\lambda}+1\right)
$$

and if strict inequality holds in either (3.10) or (3.6), then the operator $D\left(K, Z^{-1}\right)$ in (3.2) satisfies the stability condition

$$
D\left(\kappa_{1}, z^{-1}\right) \neq 0, \quad \text { for }|z| \geqslant 1 \text { except if } z=\kappa_{1}=1 \text {, and for all } \eta \Delta y .
$$

In the case $a=b,(3.10)$ is equivalent to

$$
a \leqslant \frac{1}{2} .
$$

If strict inequality holds in (3.6), then the constraints (3.10) and (3.12) can be relaxed slightly; for details, see Section 7. These constraints include the possibility of negative a or $b$. 
Theorem 2 immediately yields an analogous stability property for higher-order boundary conditions of the form (3.3).

The proofs of Theorem 1 and Lemma 2 require detailed information about the dispersion relation (2.4). This information is developed in Section 4. In Section 5 we motivate the form of $M\left(K, Z^{-1}\right)$ and prove Lemma 1 and Theorem 1 . Lemma 2 is proved in Section 6, and Theorem 2 is proved in Section 7.

We conclude this section with some remarks about the behavior of (3.9) and (3.11) for $z=\kappa_{1}=1$. In Section 2 the stability criterion (2.5) was interpreted as a solvability condition. For the boundary conditions considered here, this condition breaks down as $z \rightarrow 1$ and $\kappa_{1} \rightarrow 1$. The dispersion relation (2.4) implies that this case corresponds to $\kappa_{2}=1$ and $\eta \Delta y=0$, and (2.8) then implies that the frequency and wave numbers must be zero. A comparison with (2.10) shows that the reflection coefficient is a $0 / 0$ form in this case. For neighboring $z$ and $\kappa$ the reflection coefficient is bounded by 1 , so the breakdown in (2.5) would seem to present no difficulty.

However, this situation depends on a delicate balance between the effects of the boundary condition on incoming and outgoing waves, as the frequency tends to zero. Conceivably, this balance could be disrupted by perturbations from the ideal situation of a linear problem with constant coefficients on a uniform grid.

Another practical difficulty is illustrated by some computations described in [8]. There, some second-order $(p=2)$ boundary conditions worked well, but some third-order conditions produced large reflections that increased with time. It appears that this difficulty is due to the fact that the initial data was nonzero (but very small) at a few points within the stencil of the boundary condition, but the boundary values at $x=0$ were zero at the initial time. Thus, in effect, the boundary condition had a very small forcing term for a couple of time levels. A comparison with (2.1) and (2.10) shows that the forcing term is divided by $B\left(\kappa_{1}, z^{-1}\right)$ when the coefficient for the incoming mode is found. (Strictly speaking, this remark applies to manipulations of the Fourier-Laplace transforms of the interior scheme and boundary condition, as described at the end of Section 2.) Since the denominator tends to zero, the effect on incoming waves can be large. The actual effect on the solution depends on the order of the pole in $1 / B\left(\kappa_{1}, z^{-1}\right)$ and the number of space dimensions, since the solution can be represented by an inverse Fourier-Laplace transform. (Consider integration in polar coordinates.) The computations in question involved two space dimensions. In subsequent computations the incompatibility was removed, and third-order conditions worked better than second-order ones; the behavior of the latter was not affected by the change.

The above remarks suggest that the boundary condition (1.2) is not very robust when $p$ is sufficiently large. However, the breakdown in solvability can be relieved by replacing (1.2) with

$$
\left[\prod_{j=1}^{p}\left(\left(\cos \alpha_{j}\right) \frac{\partial}{\partial t}-c \frac{\partial}{\partial x}+\varepsilon_{j}\right)\right] u=0
$$

where the $\varepsilon_{j}$ 's are nonnegative and at least some are positive. The stability analysis of (1.2) given in Proposition 7.3 of [8] can be modified easily to analyze (3.13). If the 
discrete operator $D\left(K, Z^{-1}\right)$ is altered by adding $\varepsilon$, then the leading 1 in $M\left(K, Z^{-1}\right)$ in (3.4) is replaced by $1+\varepsilon(\Delta x / c)[a+b(\lambda / \cos \alpha)]$. The methods used to prove the above theorems can be extended easily to this case.

4. Properties of the Dispersion Relation. Here we analyze the dispersion relation (2.4),

$$
\begin{aligned}
z-2+z^{-1}= & \left(c \frac{\Delta t}{\Delta x}\right)^{2}\left(\kappa-2+\kappa^{-1}\right) \\
& +\left(c \frac{\Delta t}{\Delta y}\right)^{2}\left(e^{i \eta \Delta y}-2+e^{-i \eta \Delta y}\right) .
\end{aligned}
$$

In particular, we examine how the possible values of $\kappa$ vary as $z$ varies with $|z| \geqslant 1$ and $\eta \Delta y$ fixed. This information will be fundamental to the development in later sections. Throughout this discussion we assume that the CFL condition (3.6) is satisfied.

First consider the case of purely oscillatory waves of the form (2.7). In this case $\kappa=\exp (i \xi \Delta x)$ and $z=\exp (i \omega \Delta t)$, so (4.1) can be written as

$$
\left(\sin \frac{\omega \Delta t}{2}\right)^{2}=\left(c \frac{\Delta t}{\Delta x}\right)^{2}\left(\sin \frac{\xi \Delta x}{2}\right)^{2}+\left(c \frac{\Delta t}{\Delta y}\right)^{2}\left(\sin \frac{\eta \Delta y}{2}\right)^{2} .
$$

The graph of (4.2) is given in [8]. The group velocity associated with (2.7) is $(-\partial \omega / \partial \xi,-\partial \omega / \partial \eta)$; this vector points into (out of) the spatial domain $\Omega$ if and only if $\xi \Delta x$ and $\omega \Delta t$ have opposite (same) signs. (Here we assume $|\xi \Delta x| \leqslant \pi$, $|\omega \Delta t| \leqslant \pi$, and $|\eta \Delta y| \leqslant \pi$; this is no restriction.)

The relation (4.2) gives sufficient information about (4.1) for the analysis of the discrete conditions in [8]. However, in the present paper it is also necessary to examine (4.1) for $|z|>1$ and describe how this case relates to the case $|z|=1$.

During the discussion of the stability criterion (2.5) in Section 2, certain statements were made about the roots $\kappa_{1}$ and $\kappa_{2}$ of (4.1) corresponding to $|z| \geqslant 1$ and fixed $\eta \Delta y$. Propositions 4.1, 4.3, and 4.5 give properties of the roots $\kappa$ of (4.1) that justify those earlier statements. The precise definitions of $\kappa_{1}$ and $\kappa_{2}$ are given in the present section; no prior knowledge of the roots is assumed here. For all $z$ and $\eta \Delta y$, the two roots $\kappa$ of (4.1) are reciprocals of each other, since (4.1) is symmetric in $\kappa$ and $\kappa^{-1}$.

We begin by describing the behavior of (4.1) for $|z|>1$. Later we extend the description to $|z|=1$.

Let $D=\{z \in \mathbf{C}:|z|<1\}$ and $E=\{z \in \mathbf{C}:|z|>1\}$; and let $D_{U}, E_{U}$ and $D_{L}$, $E_{L}$ denote the intersections of $D$ and $E$ with the open upper half-plane and open lower half-plane, respectively. (See Figure 4.1(a).) Throughout this section, $\eta \Delta y$ is assumed fixed.

Proposition 4.1. If $z \in E_{U}$, then one root $\kappa$ of (4.1) lies in $D_{L}$, and the other lies in $E_{U}$. The corresponding transformations map $E_{U}$. onto $D_{L}$ and $E_{U}$ onto $E_{U}$, respectively. If $z \in E_{L}$, then one root $\kappa$ lies in $D_{U}$ and the other is in $E_{L}$; the corresponding transformations are onto.

Proof. First suppose $z \in E_{U}$. In this case, $\operatorname{Im}\left(z-2+z^{-1}\right)>0$ (see Figure 4.1(b)); a comparison with (4.1) shows $\operatorname{Im}\left(\kappa-2+\kappa^{-1}\right)>0$. It follows that the roots $\kappa$ cannot be real and cannot lie on the unit circle, since otherwise $\kappa-2+\kappa^{-1}$ 
would have to be real. Furthermore, the roots $\kappa$ are reciprocals of each other, so one root must lie inside the circle and the other must lie outside. The sign of $\operatorname{Im}\left(\kappa-2+\kappa^{-1}\right)$ then implies that the roots must lie in $D_{L}$ and $E_{U}$. To show that the maps $E_{U} \rightarrow D_{L}$ and $E_{U} \rightarrow E_{U}$ are onto, interchange the roles of $\kappa$ and $z$ in the above arguments.

The case $z \in E_{L}$ can be treated in a similar manner.

From now on, we will denote by $\kappa_{1}(z)$ and $\kappa_{2}(z)$ the roots $\kappa$ of (4.1) that lie in $D$ and $E$, respectively, when $z \in E_{U} \cup E_{L}$. These are labeled in Figure 4.1. For notational simplicity, the dependence on the parameter $\eta \Delta y$ will not be indicated explicitly.

The next proposition states some facts that will be needed later. The proposition will be used frequently without explicit reference.

Proposition 4.2. Suppose $\zeta$ is a complex number.

(a) If $\zeta$ is real, then

$$
\begin{array}{ll}
\zeta-2+\zeta^{-1}>0 & \text { if and only if } \zeta>0 \text { and } \zeta \neq 1 \\
\zeta-2+\zeta^{-1}<-4 & \text { if and only if } \zeta<0 \text { and } \zeta \neq-1 .
\end{array}
$$

(b) If $|\zeta| \neq 1$ and $\zeta+\zeta^{-1}$ is real, then $\zeta$ is real.

Proof of part (b). Suppose $|\zeta| \neq 1$ and $\zeta$ is not real. Then $\zeta$ and $\zeta^{-1}$ have different moduli and have nonzero arguments of opposite sign. Their imaginary parts thus have different magnitudes and cannot cancel.

Proposition 4.3. If $z>1$, then the roots $\kappa$ of (4.1) are real; one root lies in $(0,1)$, and the other lies in $(1,+\infty)$. If $z<-1$, then one root lies in $(-1,0)$ and the other lies in $(-\infty,-1)$. As $|z| \rightarrow+\infty$ with $z$ real, the moduli of the roots tend to 0 and $\infty$, respectively.
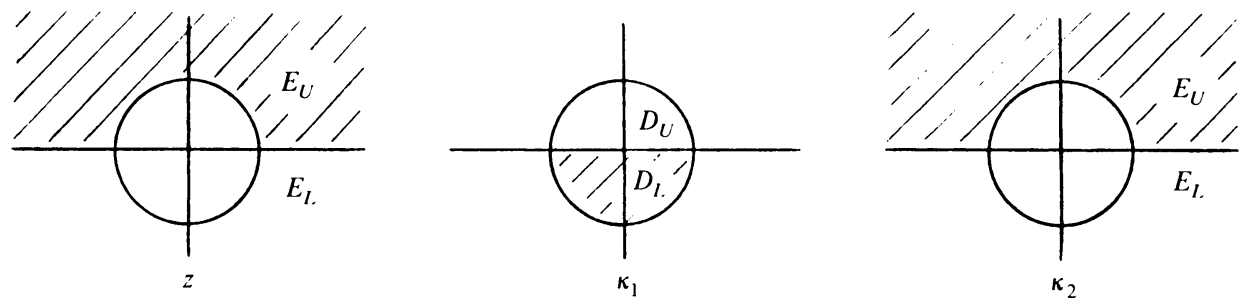

(a)

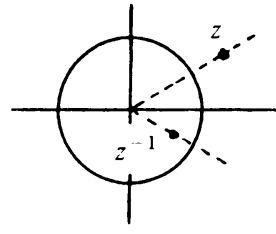

(b)

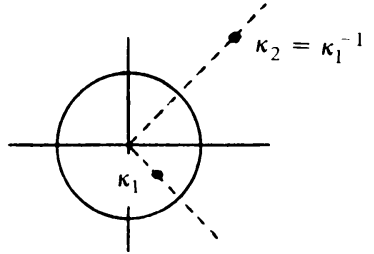

(c)

Figure 4.1

Illustration of Proposition 4.1 
Proof. First observe that $e^{i \eta \Delta y}-2+e^{-i \eta \Delta y} \leqslant 0$, with equality occurring if and only if $\eta \Delta y=0$. (Here we assume $|\eta \Delta y| \leqslant \pi$.)

If $z>1$, then the left side of (4.1) is positive. Thus $\kappa-2+\kappa^{-1}>0$, and $\kappa$ is real. The graph of $\kappa-2+\kappa^{-1}$ shows that one root $\kappa$ lies in $(0,1)$, and the other lies in $(1,+\infty)$. As $z \rightarrow+\infty$, these roots tend to 0 and $+\infty$, respectively.

If $z<-1$, then $z-2+z^{-1}<-4$. Thus $\kappa-2+\kappa^{-1}<-4$. (Here we use the CFL condition (3.6).) The remainder of the analysis is similar to that of the previous case.

For $z$ real with $|z|>1$, let $\kappa_{1}(z)$ and $\kappa_{2}(z)$ denote the roots of (4.1) so that $\left|\kappa_{1}(z)\right|<1$ and $\left|\kappa_{2}(z)\right|>1$. This notational convention is consistent with the one established after Proposition 4.1; together, the conventions define maps $\kappa_{1}: E \rightarrow D$ and $\kappa_{2}: E \rightarrow E$. The conclusions of Proposition 4.3 are illustrated as part of Figure 4.2.

Proposition 4.4. The transformations $\kappa_{1}: E \rightarrow D$ and $\kappa_{2}: E \rightarrow E$ are analytic and one-to-one.

Proof. Analyticity follows from the fact that the roots $\kappa_{1}$ and $\kappa_{2}$ are distinct. To show that the maps are one-to-one, suppose that $z_{0}$ and $z_{1}$ are distinct points in $E$ that map to the same point $\kappa$. Then for this $\kappa$, and the given $\eta \Delta y$, Eq. (4.1) has two roots $z$ outside the unit circle. This is impossible, since these roots must be reciprocals of each other.

In general, the ranges of these maps are not all of $D$ and $E$, respectively; see cases $(A)-(B)$ and $(F)-(G)$ in Figure 4.2 .

Proposition 4.5. (a) The maps $\kappa_{1}: E \rightarrow D$ and $\kappa_{2}: E \rightarrow E$ have analytic extensions to a neighborhood of any point $z_{0}$ for which $\left|z_{0}\right|=1$ and the roots $\kappa$ of (4.1) are distinct.

(b) The maps have continuous extensions to the domain $\{z \in \mathbf{C}:|z| \geqslant 1\}$.

Proof. (a) The roots are distinct when $z=z_{0}$. There thus exist analytic functions $f$ and $g$ defined on a neighborhood $Q$ of $z_{0}$ such that

(1) $f(z)$ and $g(z)$ are the roots $\kappa$ of (4.1), for each $z \in Q$; and

(2) the ranges of $f$ and $g$ are disjoint.

The functions $f$ and $g$ must then coincide with the functions $\kappa_{1}$ and $\kappa_{2}$ when $z \in Q$ and $|z|>1$.

(b) This has already been shown, except at points $z_{0}$ for which $\left|z_{0}\right|=1$ and the roots $\kappa$ of (4.1) coincide. At any such point, let $\kappa_{1}\left(z_{0}\right)$ and $\kappa_{2}\left(z_{0}\right)$ be the common value. To show continuity at $z_{0}$, use either Rouché's theorem or the continuity of the modulus of the square root.

The roots $\kappa_{1}, \kappa_{2}$ of (4.1) are distinct except when $\kappa_{1}=\kappa_{2}= \pm 1$, since $\kappa_{1} \kappa_{2}=1$.

Proposition 4.5 yields a natural definition of $\kappa_{1}$ and $\kappa_{2}$ for $|z|=1$. This is the definition that will be used from now on. Further information about these roots is given in the following proposition. It may be useful to interpret the conclusions in terms of the graph of (4.2) for $|\xi \Delta x| \leqslant \pi,|\omega \Delta t| \leqslant \pi$, and $\eta \Delta y$ fixed.

Proposition 4.6. When $|z|=1$, the dependence between $z$ and $\kappa_{1}, \kappa_{2}$ is that which is indicated in Figure 4.2. The figure illustrates the case where $0 \leqslant \arg z \leqslant \pi$ when $|z|=1$. If $-\pi \leqslant \arg z \leqslant 0$ when $|z|=1$, then the signs of $\arg \kappa_{1}$ and $\arg \kappa_{2}$ should be reversed. Here $\eta \Delta y$ is fixed. 


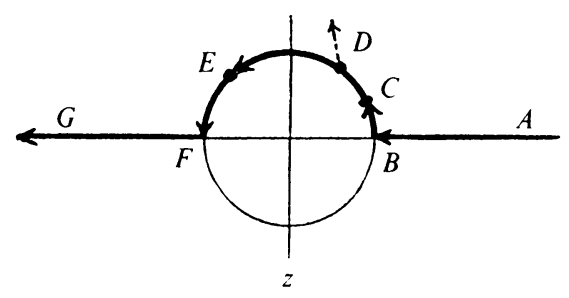

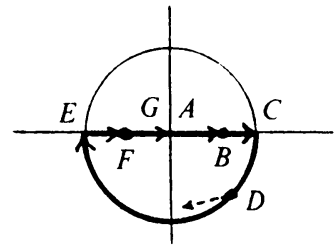

$\kappa_{1}$ (incoming)

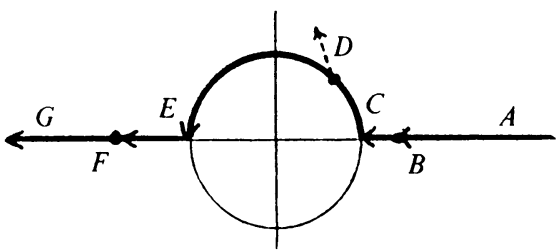

$\kappa_{2}$ (outgoing)

FigURE 4.2

Illustration of Propositions 4.3 and 4.6. Letters indicate corresponding values. Arrows indicate directions of relative movement. In this picture $\eta \Delta y \neq 0$, and $0 \leqslant \arg z \leqslant \pi$ when $|z|=1$.

Proof. Suppose $z=1$. If $\eta \Delta y \neq 0$, then $\kappa-2+\kappa^{-1}$ is positive, and $\kappa_{1}<1<\kappa_{2}$. This is case $(B)$ in the figure.

For the moment, continue to assume $\eta \Delta y \neq 0$, and move $z$ around the unit circle. The left side of (4.1) becomes negative. At some point, $\kappa-2+\kappa^{-1}=0$, so that $\kappa_{1}$ and $\kappa_{2}$ come together to form a double root $\kappa_{1}=\kappa_{2}=1$. This is case $(C)$ in the figure. If $\eta \Delta y=0$, then this coalescence occurs when $z=1$. Between cases $(B)$ and $(C)$, the modes (2.3) are evanescent.

Now move $z$ further around the circle. Then $z-2+z^{-1}$ becomes more negative, and $\kappa-2+\kappa^{-1}<0$. The two values of $\kappa$ must then lie on the unit circle; this follows from parts (a) and (b) of Proposition 4.2. The labeling of $\kappa_{1}$ and $\kappa_{2}$ in the figure follows from Propositions 4.1 and 4.5.

The remainder of the analysis is similar to the above.

According to (4.2) and related discussion, a purely oscillatory mode has incoming (outgoing) group velocity if and only if $\arg z$ and $\arg \kappa$ have opposite (same) signs. Thus $\kappa_{1}$ and $\kappa_{2}$ are associated with incoming and outgoing modes, respectively.

A related identification of these modes is given by the following. Consider a point $z$ for which $|z|=\left|\kappa_{1}(z)\right|=\left|\kappa_{2}(z)\right|=1$, with $\kappa_{1} \neq \kappa_{2}$. If $z$ is perturbed so that $|z|>1$, then $\kappa_{1}$ and $\kappa_{2}$ are perturbed so that $\left|\kappa_{1}\right|<1$ and $\left|\kappa_{2}\right|>1$. This is indicated by the dotted arrows at the positions $(D)$ in Figure 4.3. Thus incoming waves (subscript 1) are associated with perturbations to modes (2.3), $\kappa^{j} e^{i \eta y_{z}}{ }^{n}$, that decay as $x$ increases, when $|z|>1$. Outgoing waves are associated with modes that decay as $x$ decreases.

This is an example of a labeling process that is found more generally; see Trefethen [10]. (Also see [7].) In the proof of the general case one uses conformal dependence of $\kappa$ on $z$. 
5. Properties of $M\left(K, Z^{-1}\right)$. The operator $M\left(K, Z^{-1}\right)$ was defined in (3.4) and (3.5). Here we motivate its form and prove Lemma 1 and Theorem 1.

The operator will be derived without reference to discretizations of $(\cos \alpha) \partial / \partial t-$ $c \partial / \partial x$. Instead, we work directly with properties of the interior difference scheme to produce an operator that defines a discrete absorbing boundary condition. The operator can be adjusted so that it does its best absorption for waves traveling at angle of incidence $\pm \alpha$. (At this point we refer to waves that are resolved well by the grid.) After the operator is derived, we show that it is equivalent to certain discretizations of $(\cos \alpha) \partial / \partial t-c \partial / \partial x$. This is Lemma 1 .

The operator also has a desirable absorption property for all oscillatory waves admitted by the interior difference scheme, not just those that are resolved well by the grid. This is given in Theorem 1.

5.1. Construction of $M\left(K, Z^{-1}\right)$. In order to motivate the form of $M\left(K, Z^{-1}\right)$, we begin with a description of the space-time extrapolation boundary conditions discussed in [8]. These use powers of the operator

$$
S\left(K, Z^{-1}\right)=I-Z^{-1} K
$$

The reflection coefficient for the first-order version is

$$
R_{S}=-\frac{S\left(\kappa_{2}, z^{-1}\right)}{S\left(\kappa_{1}, z^{-1}\right)}
$$

In the dispersion relation (4.1) assume $\eta \Delta y \neq 0$. Thus $z \neq 1$ when $\kappa_{1}=\kappa_{2}=1$. (See case $(C)$ in Figure 4.2.) If $z$ is moved around the unit circle away from 1, then $\kappa_{1}$ and $\kappa_{2}$ also move around the circle. The $\kappa$ 's move more rapidly than does $z$, since each $\kappa$ moves through a complete semicircle when $z$ moves through part of a semicircle. Thus at some point, $\kappa_{2}$ catches up with $z$. At this point, $1-z^{-1} \kappa_{2}=0$, and $R_{S}=0$. Since $z=\exp (i \omega \Delta t)$ and $\kappa=\exp (i \xi \Delta x), z=\kappa_{2}$ implies $\omega \Delta t=\xi_{2} \Delta x$. But $c \xi_{2} / \omega \approx \cos \theta$ for waves that are resolved well by the grid, where $\theta$ is the angle of incidence. (See Figure 4.3 in [8], or calculate group velocity.) The best absorption then occurs for $\cos \theta \approx c \Delta t / \Delta x=\lambda$.

The operator in (5.1) is equivalent to certain discretizations of $\lambda \partial / \partial t-c \partial / \partial x$. Two examples are given in Subsection 5.2.

The angle of best absorption for (5.1) is determined by the point where $\kappa_{2}$ catches up with $z$. Now suppose we want a boundary condition that is tuned to a different angle of incidence. To that end, consider the more general operator

$$
M\left(K, Z^{-1}\right)=I-P\left(Z^{-1}\right) Q(K),
$$

where $P$ and $Q$ are linear polynomials having real coefficients. $P$ and $Q$ will be chosen so that $\arg Q\left(\kappa_{2}\right)$ coincides with $\arg P(z)$ at frequencies corresponding to the desired angle.

The following construction accomplishes this goal. Let

$$
P\left(z^{-1}\right)=1+r_{Z}\left(z^{-1}-1\right), \quad Q(\kappa)=1+r_{K}(\kappa-1),
$$

where $r_{Z}$ and $r_{K}$ are real nonzero constants that have the same sign. When (5.3) is inserted into (5.2), the result is the operator $M\left(K, Z^{-1}\right)$ defined in (3.4) and (3.5). 

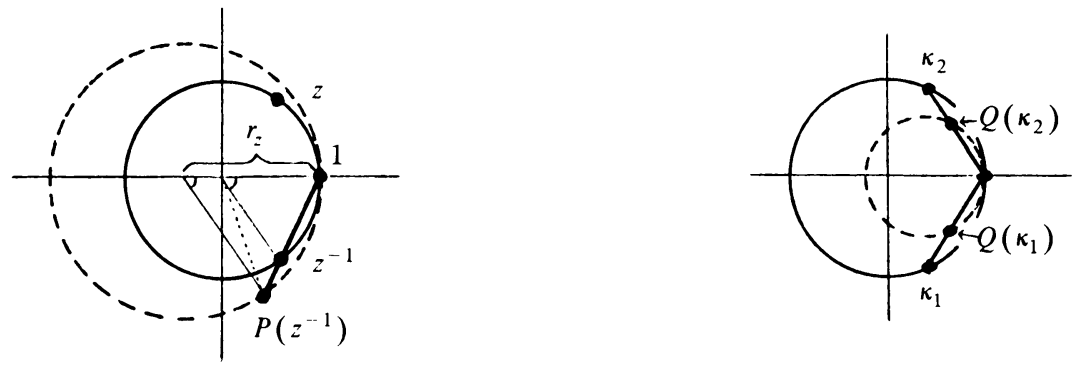

Figure 5.1

Geometrical representations of $P\left(z^{-1}\right)$ and $Q\left(\kappa_{j}\right)$ for $|z|=\left|\kappa_{j}\right|=1$. In these pictures, $r_{Z}>1$, and $0<r_{K}<1$.

$P$ and $Q$ map the unit circle to circles of radius $\left|r_{Z}\right|$ and $\left|r_{K}\right|$, respectively, with $P(1)=Q(1)=1$. A geometrical interpretation of $P\left(z^{-1}\right)$ and $Q(\kappa)$ follows from the fact that $P\left(z^{-1}\right)-1$ and $Q(\kappa)-1$ are real multiples of $z^{-1}-1$ and $\kappa-1$, respectively. (See Figure 5.1.) In the figure, $r_{Z}>1$ and $0<r_{K}<1$. In each graph the solid circle is the unit circle.

The reflection coefficient for $M\left(K, Z^{-1}\right)$ is

$$
R_{M}=-\frac{1-P\left(z^{-1}\right) Q\left(\kappa_{2}\right)}{1-P\left(z^{-1}\right) Q\left(\kappa_{1}\right)} .
$$

Now consider the angles of best absorption for oscillatory waves that are resolved well by the grid. For such waves, $\omega \Delta t$ and $\xi \Delta x$ are small, so $z$ and $\kappa$ are close to 1 . In the following discussion, we assume that $r_{Z}$ and $r_{K}$ are positive; a related analysis applies to the case where they are both negative.

First locate $P\left(z^{-1}\right)$ and $Q(\kappa)$. Figure 5.1 implies that $r_{Z} \arg z^{-1}$ is the (signed) arc length from 1 to $P\left(z^{-1}\right)$ along the circle of radius $r_{Z}$. Similarly, $\arg P\left(z^{-1}\right)$ is the (signed) arc length along the unit circle to the point whose argument is that of $P\left(z^{-1}\right)$. If $z$ is near 1 these lengths coincide to leading order, so $r_{Z} \arg z^{-1} \approx$ $\arg P\left(z^{-1}\right)$. Similarly, when $\kappa$ is near 1 , we have $r_{K} \arg \kappa \approx \arg Q(\kappa)$.

In these cases, $P\left(z^{-1}\right)$ and $Q(\kappa)$ have moduli nearly equal to 1 . Thus if

$$
\arg P\left(z^{-1}\right) \approx-\arg Q\left(\kappa_{2}\right),
$$

then

$$
\left|1-P\left(z^{-1}\right) Q\left(\kappa_{2}\right)\right| \ll\left|1-P\left(z^{-1}\right) Q\left(\kappa_{1}\right)\right|,
$$

and $R_{M}$ is essentially zero. But (5.5) means $r_{Z} \arg z^{-1} \approx-r_{K} \arg \kappa_{2}$, so

$$
c \frac{\xi_{2}}{\omega} \approx \frac{r_{Z}}{r_{K}} \frac{c \Delta t}{\Delta x} \text {. }
$$

This implies that the reflection is essentially zero for waves traveling at angles of incidence $\theta$ for which $(\cos \theta) / \lambda \approx r_{Z} / r_{K}$. The boundary condition can therefore be adjusted to an arbitrary angle $\alpha$ by choosing $r_{7}$ and $r_{K}$ so that

$$
\frac{r_{Z}}{r_{K}}=\frac{\cos \alpha}{\lambda}
$$


Taylor expansions of the exponentials in (5.4) imply that, to leading order, $-R_{M}$ has the form of a factor in (1.3). The above discussion applies only to those oscillatory waves that are resolved well by the grid; for general reflection properties, see Theorem 1.

Compositions of operators of the form $M\left(K, Z^{-1}\right)$, analogous to (3.3), can be used to yield higher-order absorption. (Cf. (2.11).)

5.2. Proof of Lemma 1; Examples. Here we show that $M\left(K, Z^{-1}\right)$ provides a representation of the difference operator $D\left(K, Z^{-1}\right)$ defined in (3.2).

The definition (3.4) of $M\left(K, Z^{-1}\right)$ implies

$$
-M\left(K, Z^{-1}\right)=r_{Z}\left(Z^{-1}-I\right)+r_{K}(K-I)+r_{Z} r_{K}\left(Z^{-1}-I\right)(K-I) .
$$

A manipulation of $D\left(K, Z^{-1}\right)$ yields

$$
\begin{aligned}
-\frac{\Delta x}{c} D\left(K, Z^{-1}\right)= & \left(\frac{\cos \alpha}{\lambda}\right)\left(Z^{-1}-I\right)+(K-I) \\
& +\left[a\left(\frac{\cos \alpha}{\lambda}\right)+b\right]\left(Z^{-1}-I\right)(K-I) .
\end{aligned}
$$

When (5.9) is multiplied by

$$
\left(\frac{\lambda}{\cos \alpha}\right)\left[a\left(\frac{\cos \alpha}{\lambda}\right)+b\right]
$$

the right side is the same as the right side of (5.8), with

$$
r_{Z}=a\left(\frac{\cos \alpha}{\lambda}\right)+b, \quad r_{K}=a+b\left(\frac{\lambda}{\cos \alpha}\right)
$$

Thus,

$$
\frac{\Delta x}{c}\left[a+b\left(\frac{\lambda}{\cos \alpha}\right)\right] D\left(K, Z^{-1}\right)=M\left(K, Z^{-1}\right) .
$$

This completes the proof.

The parameters defined in (5.10) satisfy the constraint (5.7). We now give some specific approximations to (3.1) that are included in the general form $D\left(K, Z^{-1}\right)$.

(a) Forward Euler: $a=0, b=1$. The stencil has an " $L$ " shape. Here $r_{Z}=1$ and $r_{K}=\lambda / \cos \alpha$.

(b) Backward Euler: $a=b=0$. The stencil has inverted " $L$ " shape. In this case, $M\left(K, Z^{-1}\right)$ cannot be used to give a representation of $D\left(K, Z^{-1}\right)$. However, stability and reflection properties for this case will be handled easily by separate arguments.

(c) Box scheme: $a=b=1 / 2$. In this case, $r_{Z}<1<r_{K}$ or $r_{K}<1<r_{Z}$, unless $\lambda=\cos \alpha$.

The stability properties of these examples are summarized in Section 7.

After Lemma 1 was stated in Section 3, it was pointed out that different pairs $(a, b)$ can lead to equivalent operators $D\left(K, Z^{-1}\right)$. An example of this is given by the space-time extrapolation operator (5.1); this is equivalent to both the forward Euler and box scheme approximations to $\lambda \partial / \partial t-c \partial / \partial x$.

5.3. Proof of Theorem 1 (Reflection Property). Here we show

$$
\left|\frac{D\left(\kappa_{2}, z^{-1}\right)}{D\left(\kappa_{1}, z^{-1}\right)}\right|<1
$$


when $|z|=\left|\kappa_{1}\right|=\left|\kappa_{2}\right|=1$ and $\kappa_{1} \neq \kappa_{2}$. The dispersion relation (4.1) implies $\kappa_{1} \kappa_{2}=1$, so $\kappa_{1}$ and $\kappa_{2}$ coincide only when $\kappa_{1}=\kappa_{2}= \pm 1$. Except when $z=\kappa_{1}=\kappa_{2}=1$, this case corresponds to zero normal group velocity; this can be determined from the graph of (4.2) for fixed $\eta \Delta y$ (see [8]), or from the group velocity $(-\partial \omega / \partial \xi$, $-\partial \omega / \partial \eta)$.

If $z=\kappa_{1}=\kappa_{2}=1$ then $\eta \Delta y=0$ (see (4.1)), and the mode (2.3) corresponds to frequency zero. In this case the behavior of neighboring frequencies is described by the consistency of $D\left(K, Z^{-1}\right)$ with (3.1).

If $z, \kappa_{1}$, and $\kappa_{2}$ lie on the unit circle with $\kappa_{1} \neq \kappa_{2}$, then these numbers cannot be real. This is implied by Figure 4.2.

In order to prove (5.12), we need to consider two different cases.

Case $\mathrm{I}: a+b(\lambda / \cos \alpha) \neq 0$. In this case (5.12) is equivalent to

$$
\left|\frac{M\left(\kappa_{2}, z^{-1}\right)}{M\left(\kappa_{1}, z^{-1}\right)}\right|<1
$$

(see (5.11)), or $\left|R_{M}\right|<1$. The formulas in (5.10) imply that $r_{Z}$ and $r_{K}$ have the same sign.

Consider the factors $P\left(Z^{-1}\right)$ and $Q(K)$ in $M\left(K, Z^{-1}\right) . P\left(z^{-1}\right), Q\left(\kappa_{1}\right)$ and $Q\left(\kappa_{2}\right)$ are not real, since $z^{-1}, \kappa_{1}$, and $\kappa_{2}$ are not real. Figure 5.1 shows that $\arg P\left(z^{-1}\right)$ and $\arg Q\left(\kappa_{1}\right)$ have the same sign, whereas $\arg P\left(z^{-1}\right)$ and $\arg Q\left(\kappa_{2}\right)$ have opposite signs. Furthermore, $\left|Q\left(\kappa_{1}\right)\right|=\left|Q\left(\kappa_{2}\right)\right|$ and $\arg Q\left(\kappa_{1}\right)=-\arg Q\left(\kappa_{2}\right)$. The line through $P\left(z^{-1}\right)$ and the origin is therefore the set of all points in the complex plane that are equidistant from $P\left(z^{-1}\right) Q\left(\kappa_{1}\right)$ and $P\left(z^{-1}\right) Q\left(\kappa_{2}\right)$. (See Figure 5.2(a).) $P\left(z^{-1}\right) Q\left(\kappa_{2}\right)$ lies on the same side of this line as 1. A comparison with (5.4) and (5.2) yields (5.13).

Case II: $a+b(\lambda / \cos \alpha)=0$. In this case $M\left(K, Z^{-1}\right)$ cannot be used to represent $D\left(K, Z^{-1}\right)$. However, (5.9) now reduces to the simplified form

$$
-\frac{\Delta x}{c} D\left(K, Z^{-1}\right)=\left(\frac{\cos \alpha}{\lambda}\right)\left(Z^{-1}-I\right)+(K-I) \text {. }
$$

A typical configuration of $z^{-1}-1$ and $\kappa_{j}-1$ is given in Figure 5.2(b). Clearly $\left|D\left(\kappa_{2}, z^{-1}\right)\right|<\left|D\left(\kappa_{1}, z^{-1}\right)\right|$, and (5.12) follows.

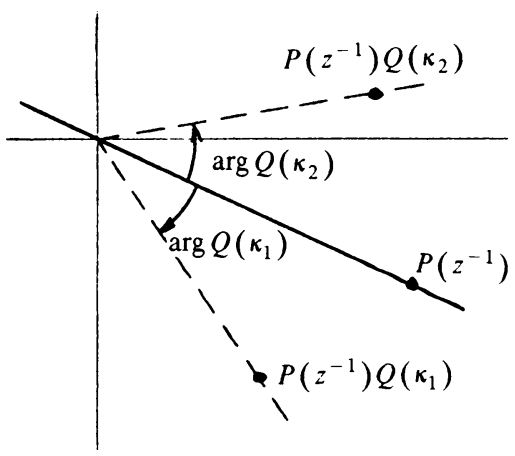

(a) Case I

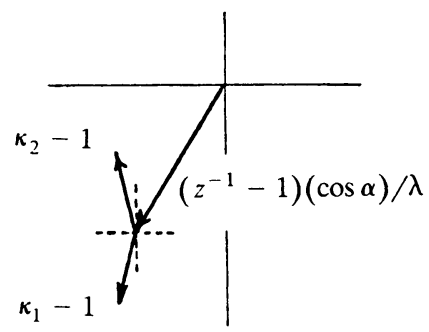

(b) Case II

FIGURE 5.2

Illustration of Theorem 1 . 
6. Proof of Lemma 2 (Stability for $M\left(K, Z^{-1}\right)$ ). Here we show $M\left(\kappa_{1}, z^{-1}\right) \neq 0$ for $|z| \geqslant 1$ except when $z=\kappa_{1}=1$, subject to the conditions described in the theorem. Equation (5.2) shows that the desired conclusion is equivalent to the statement

$$
P\left(z^{-1}\right) Q\left(\kappa_{1}\right) \neq 1 \text { for }|z| \geqslant 1 \text {, except when } z=\kappa_{1}=1 \text {, and for all } \eta \Delta y .
$$

It is assumed that $r_{Z}$ and $r_{K}$ have the same sign. (This is no restriction.)

First consider the special case where $0<r_{Z}<1$ and $0<r_{K}<1$. This case is covered by the general analysis given below, but here it is possible to use a special argument that is much simpler than the general one. In this argument, the only fact about the dispersion relation that is used is that $\left|\kappa_{1}\right| \leqslant 1$ when $|z| \geqslant 1$; this suggests the possibility of easily extending this method to other interior schemes or other problems.

If $0<r_{Z}<1$, then the formula

$$
P\left(z^{-1}\right)-1=r_{Z}\left(z^{-1}-1\right)
$$

(see (5.3)) implies that the map $z^{-1} \mapsto P\left(z^{-1}\right)$ is a contraction toward 1, as indicated in Figure 6.1. In each graph the shaded area is the domain of possible values of the specified quantity. $P(1)=1$, but otherwise $\left|P\left(z^{-1}\right)\right|<1$ for $|z| \geqslant 1$. An analogous conclusion holds for $Q\left(\kappa_{1}\right)$, since $\left|\kappa_{1}\right| \leqslant 1$. Thus $\left|P\left(z^{-1}\right) Q\left(\kappa_{1}\right)\right|<1$ for $|z| \geqslant 1$, except when $z=\kappa_{1}=1$, and (6.1) follows.

Similar analyses hold if $0<r_{Z}<1$ and $r_{K}=1$, or if $r_{Z}$ and $r_{K}$ are both negative. In the latter case, (6.2) implies that $P\left(z^{-1}\right)$ lies outside the unit circle if $|z| \geqslant 1$ and $z \neq 1$. A similar conclusion applies to $Q\left(\kappa_{1}\right)$, so $\left|P\left(z^{-1}\right) Q\left(\kappa_{1}\right)\right|>1$ except when $z=\kappa_{1}=1$.

The above arguments fail for other choices of $r_{Z}$ and $r_{K}$. In such cases it is possible to have $\left|P\left(z^{-1}\right) Q\left(\kappa_{1}\right)\right|=1$ in situations other than $z=\kappa_{1}=1$. These cases are of interest; for example, the box scheme can yield $r_{Z}>1$, or $r_{K}>1$, or $r_{Z}=r_{K}=1$. (See Subsection 5.2.) A more careful analysis is therefore needed. In the following discussion we assume $\eta \Delta y$ is fixed, $r_{Z}>0$, and $r_{K}>0$.

Case I: $|z| \geqslant 1$, $z$ not real. First suppose $z \in E_{U}$. Figure 4.1 shows $z^{-1} \in D_{L}$ and $\kappa_{1} \in D_{L}$ in this case. The geometrical interpretations of $P\left(z^{-1}\right)$ and $Q\left(\kappa_{1}\right)$ imply that $P\left(z^{-1}\right)$ and $Q\left(\kappa_{1}\right)$ must then lie in the lower half-plane. (Cf. Figure 5.1.) Since their arguments are between $-\pi$ and 0 , their product cannot be real and positive. In particular, $P\left(z^{-1}\right) Q\left(\kappa_{1}\right) \neq 1$, which is the desired conclusion.

Next suppose $|z|=1$ and $\operatorname{Im} z>0$. Then $z^{-1}$ lies on the lower unit semicircle, and $P\left(z^{-1}\right)$ is in the lower half-plane. Cases $(B)-(F)$ in Figure 4.2 show that $\kappa_{1}$ lies either on the lower unit semicircle or on the real axis. Thus $Q\left(\kappa_{1}\right)$ is either in the

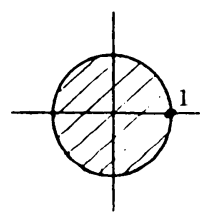

$z^{-1}$

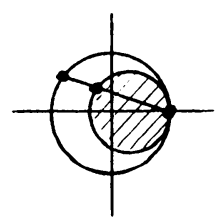

$P\left(z^{-1}\right)$

Figure 6.1

$P\left(z^{-1}\right)$ for $|z| \geqslant 1$ and $0<r_{Z}<1$. 

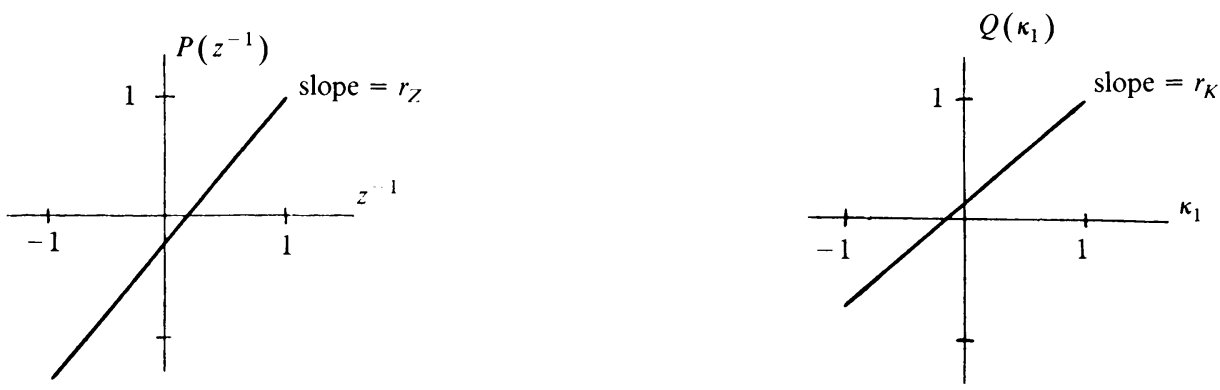

FIGURE 6.2

Values of $P\left(z^{-1}\right)$ and $Q\left(\kappa_{1}\right)$ for $|z| \geqslant 1$, with $z$ real.

lower half-plane or on the real axis. In the first case, $P\left(z^{-1}\right) Q\left(\kappa_{1}\right)$ cannot be real and positive, so $P\left(z^{-1}\right) Q\left(\kappa_{1}\right) \neq 1$. In the second case, $P\left(z^{-1}\right) Q\left(\kappa_{1}\right)$ can be real only if $Q\left(\kappa_{1}\right)=0$. But then the product would be zero, and we still have $P\left(z^{-1}\right) Q\left(\kappa_{1}\right) \neq 1$.

Therefore, $P\left(z^{-1}\right) Q\left(\kappa_{1}\right) \neq 1$ if $|z| \geqslant 1$ and $\operatorname{Im} z>0$. A similar analysis applies to the case $\operatorname{Im} z<0$.

Case II: $|z| \geqslant 1$ with $z$ real. This is the only case that can yield instability, and it is the only source of restrictions on $r_{Z}$ and $r_{K}$.

Here $-1 \leqslant z^{-1} \leqslant 1$. Figure 4.2 shows that $\kappa_{1}$ also lies in $[-1,1]$, and, in fact, is often restricted to a proper subinterval of $[-1,1]$. The corresponding values of $P\left(z^{-1}\right)$ and $Q\left(\kappa_{1}\right)$ are graphed in Figure 6.2. (Cf. (5.3).) On the given domains, $P\left(z^{-1}\right)<1$ except if $z=1$, and $Q\left(\kappa_{1}\right)<1$ except if $\kappa_{1}=1$. Thus the only way to have $P\left(z^{-1}\right) Q\left(\kappa_{1}\right)=1$ (except when $z=\kappa_{1}=1$ ) is for $P\left(z^{-1}\right)$ and $Q\left(\kappa_{1}\right)$ both to be negative.

We now obtain the sufficient condition (3.8) given in Lemma 2. If $P(-1) \geqslant 0$, then $P\left(z^{-1}\right)>0$ whenever $-1<z^{-1} \leqslant 1$; (6.1) must then hold, regardless of the behavior of $Q$. Similarly, if $Q(-1) \geqslant 0$, then (6.1) holds, regardless of the behavior of $P$. Thus (6.1) can fail only if $P(-1)<0$ and $Q(-1)<0$. So suppose that $P(-1)$ and $Q(-1)$ are negative, and now also assume $P(-1) Q(-1) \leqslant 1$. Then for any $z^{-1}$ and $\kappa_{1}$ in $[-1,1]$ for which $P\left(z^{-1}\right)<0$ and $Q\left(\kappa_{1}\right)<0$, we must have $P\left(z^{-1}\right) Q\left(\kappa_{1}\right)$ $<1$, except if $P(-1) Q(-1)=1$ and $z=\kappa_{1}=-1$. (This follows from the signs and monotonicity of $P$ and $Q$.) For any other $z^{-1}$ and $\kappa_{1}$ in $[-1,1]$, it has already been established that $P\left(z^{-1}\right) Q\left(\kappa_{1}\right) \neq 1$. Thus for the case where $P(-1)$ and $Q(-1)$ are negative, the stability condition (6.1) is satisfied if

$$
P(-1) Q(-1) \leqslant 1
$$

and if either strict inequality holds in (6.3) or it is not possible to have solutions of the dispersion relation for which $z=\kappa_{1}=-1$. The ideas behind Propositions 4.3 and 4.6 show that $z=\kappa_{1}=-1$ is impossible if and only if strict inequality holds in the CFL condition (3.6). Thus, for the case where $P(-1)<0$ and $Q(-1)<0$, the stability condition (6.1) is satisfied if (6.3) holds and if strict inequality holds in either (6.3) or (3.6).

We now describe the above conclusions in terms of conditions on $r_{Z}$ and $r_{K}$. Throughout this discussion we have assumed $r_{Z}>0$ and $r_{K}>0$. The case where $P(-1) \geqslant 0$ and $Q(-1)$ is unrestricted thus corresponds to $0<r_{Z} \leqslant 1 / 2$ and 
$r_{K}>0$. The case $Q(-1) \geqslant 0$ corresponds to $0<r_{K} \leqslant 1 / 2$ and $r_{Z}>0$. In the case where $P(-1)$ and $Q(-1)$ are negative, we have $r_{Z}>1 / 2$ and $r_{K}>1 / 2$. The formulas (5.3) for $P\left(z^{-1}\right)$ and $Q(\kappa)$ show that (6.3) is equivalent to

$$
\left(2 r_{Z}-1\right)\left(2 r_{K}-1\right) \leqslant 1 .
$$

The union of the sets of all $\left(r_{Z}, r_{K}\right)$ described here is defined by (6.4) and the constraints $r_{Z}>0$ and $r_{K}>0$; consider the region in the $\left(r_{Z}, r_{K}\right)$-plane bounded by the positive coordinate axes and the hyperbola $\left(2 r_{Z}-1\right)\left(2 r_{K}-1\right)=1$. The desired sufficient condition (3.8) has thus been established.

If strict inequality holds in the CFL condition (3.6), then the constraints imposed by (3.8) can be relaxed slightly. The analysis is similar to the above, but more tedious, so here we merely outline the results.

If strict inequality holds in (3.6), then $\kappa_{1}$ can never equal -1 for $z \leqslant-1$. An analysis of the dispersion relation (4.1) shows that the most negative $\kappa_{1}$ (for $z \leqslant-1$ ) occurs when $z=-1$ and $\eta \Delta y= \pm \pi$. The extreme $\kappa_{1}$ is $-d+\left(d^{2}-1\right)^{1 / 2}$, where

$$
d=-1+2\left(1-\left(c \frac{\Delta t}{\Delta y}\right)^{2}\right) /\left(c \frac{\Delta t}{\Delta x}\right)^{2} .
$$

(The CFL condition (3.6) is satisfied if and only if $d \geqslant 1$.) An analysis similar to the above shows that (6.4) can be replaced by

$$
\left(2 r_{Z}-1\right)\left[\left(1+d-\left(d^{2}-1\right)^{1 / 2}\right) r_{K}-1\right]<1 .
$$

A study of hyperbolas in the $\left(r_{Z}, r_{K}\right)$-plane shows that (6.6) represents a loosening of the restriction (6.4).

The inequality (6.6) is necessary and sufficient for the stability condition (6.1) to hold, except for the following technicality. If $P(0)<0, Q(0)<0$, and $P(0) Q(0)=1$, then $P\left(z^{-1}\right) Q\left(\kappa_{1}\right)>1$ for $z<-1$ and $P\left(z^{-1}\right) Q\left(\kappa_{1}\right)<1$ for $z>1$. However, this situation is sensitive to perturbations in $r_{Z}$ and $r_{K}$. This case is of little practical significance, since (3.5) shows that $r_{Z}$ and $r_{K}$ depend on the mesh ratio and wave speed. The definition (3.4) of $M\left(K, Z^{-1}\right)$ shows that $P(0) Q(0)=1$ if and only if $M\left(\kappa_{1}, z^{-1}\right) \rightarrow 0$ as $|z| \rightarrow \infty$ and $\left|\kappa_{1}\right| \rightarrow 0$. This situation could therefore be avoided by requiring uniformity in (3.9) and (6.1) as $|z| \rightarrow \infty$.

7. Proof of Theorem 2 (Stability for $D\left(K, Z^{-1}\right)$ ). The goal is to show

$$
D\left(\kappa_{1}, z^{-1}\right) \neq 0, \quad \text { for }|z| \geqslant 1 \text { except if } z=\kappa_{1}=1 \text {, and for all } \eta \Delta y,
$$

subject to the conditions described in the theorem. This conclusion immediately yields an analogous stability property for the general boundary condition (3.3).

Lemma 1 states that if $a+b(\lambda / \cos \alpha)$ is nonzero, then $D\left(K, Z^{-1}\right)$ is a nonzero multiple of $M\left(K, Z^{-1}\right)$, with

$$
r_{Z}=a\left(\frac{\cos \alpha}{\lambda}\right)+b ; \quad r_{K}=\left(\frac{\lambda}{\cos \alpha}\right) r_{Z}
$$

In this case, (7.1) is equivalent to the statement (3.9) that is obtained by replacing $D\left(\kappa_{1}, z^{-1}\right)$ with $M\left(\kappa_{1}, z^{-1}\right)$. The analysis then amounts to an application of Lemma 2. The case $a+b(\lambda / \cos \alpha)=0$ will be handled separately. 
Lemma 2 gives separate descriptions of the cases where $r_{Z}$ and $r_{K}$ are both positive and both negative. Therefore, we finally divide the proof of Theorem 2 into the following cases:

Case I: $a+b(\lambda / \cos \alpha)<0$. Here $r_{Z}$ and $r_{K}$ are negative, and Lemma 2 implies that (3.9) and (7.1) hold.

Case II: $a+b(\lambda / \cos \alpha)=0$. Here $M\left(K, Z^{-1}\right)$ cannot be used to represent $D\left(K, Z^{-1}\right)$. However, (5.9) implies

$$
-\frac{\Delta x}{c} D\left(\kappa, z^{-1}\right)=\left(\frac{\cos \alpha}{\lambda}\right)\left(z^{-1}-1\right)+(\kappa-1)
$$

in this case. If $|z| \geqslant 1$, then $z^{-1}-1$ has negative real part except when $z=1$. Furthermore, $\left|\kappa_{1}\right| \leqslant 1$, so $\kappa_{1}-1$ has negative real part except when $\kappa_{1}=1$. Since $|\alpha|<\pi / 2$, we can conclude $D\left(\kappa_{1}, z^{-1}\right) \neq 0$ except when $z=\kappa_{1}=1$. This is (7.1).

Case III: $a+b(\lambda / \cos \alpha)>0$. Here Lemma 2 can be applied, with $r_{Z}>0$ and $r_{K}>0$. For this case, Lemma 2 states that (3.9) (and (7.1)) must hold if

$$
\left(2 r_{Z}-1\right)\left(2 r_{K}-1\right) \leqslant 1
$$

and if strict inequality holds either in (7.3) or in the CFL condition (3.6). When (7.2) is substituted into (7.3), the result is

$$
a\left(\frac{\cos \alpha}{\lambda}\right)+b \leqslant \frac{1}{2}\left(\frac{\cos \alpha}{\lambda}+1\right)
$$

Cases I-III then imply that if (7.4) holds, and if strict inequality holds in (7.4) or (3.6), then the stability condition (7.1) must hold. This completes the proof of the part of Theorem 2 associated with (3.10) and (3.12).

Some numerical computations described in Section 8 suggest that Case III is the case of greatest interest in practice.

The above analysis gives a condition that is sufficient for (7.1) to hold. We next apply the condition (6.6) that is essentially necessary and sufficient for the stability condition to hold for $M\left(K, Z^{-1}\right)$, when $r_{Z}$ and $r_{K}$ are positive. This condition is equivalent to

$$
a\left(\frac{\cos \alpha}{\lambda}\right)+b<\frac{1}{\beta}\left(\frac{\cos \alpha}{\lambda}\right)+\frac{1}{2} .
$$

Here $\beta=1+d-\left(d^{2}-1\right)^{1 / 2}$, where $d$ is given in (6.5). The CFL condition (3.6) is satisfied if and only if $\beta \leqslant 2$.

This completes the proof of Theorem 2 .

We conclude with some examples. According to remarks made after Lemma 1 in Section 3, every operator $D\left(K, Z^{-1}\right)$ of the form (3.2) is equivalent to an operator of that form for which $a=b$. For this case (7.4) says $a \leqslant 1 / 2$.

The general necessary and sufficient condition (7.5) involves $\alpha, \lambda=c \Delta t / \Delta x$, and $c \Delta t / \Delta y$. If $\Delta x=\Delta y$, then the CFL condition (3.6) implies $\lambda \leqslant 2^{-1 / 2} \doteq .707$. (This assumes two space dimensions.) In the numerical computations described in Section 8 , we use $\Delta x=\Delta y$ and $\lambda=.625$, and in most cases we assume $a=b$. Under these assumptions, (7.5) says $a<.684$ when $\alpha=0$; if $\alpha=\pi / 6$, then (7.5) says $a<.674$. The right side of (7.5) approaches $1 / 2$ as $|\alpha| \rightarrow \pi / 2$. 
Next consider the specific discretizations mentioned in Subsection 5.2.

(a) Forward Euler: $a=0, b=1$. In this case (7.4) is equivalent to $\cos \alpha \geqslant \lambda$. The necessary and sufficient condition (7.5) says $\cos \alpha>\lambda(\beta / 2)$.

(b) Backward Euler: $a=b=0$. This is Case II discussed above. For this example no restriction is placed on $\alpha$ or on the mesh, except that the CFL condition (3.6) must be satisfied, and we need $|\alpha|<\pi / 2$. The stability analysis of this case depends only on the fact that $\left|\kappa_{1}\right| \leqslant 1$ when $|z| \geqslant 1$, and it thus may extend easily to other interior schemes or other problems.

(c) Box scheme: $a=b=1 / 2$. In this case the stability condition (7.1) holds whenever strict inequality holds in the CFL condition.

8. Numerical Computations. Here we give the results of some numerical computations involving the boundary conditions described in this paper. We present separate series of tests which

(1) illustrate the stability limit (7.5),

(2) demonstrate how the amount of reflection varies with the choice of $\alpha_{1}$ and $\alpha_{2}$ in (1.2), and

(3) illustrate the behavior of the boundary conditions near a corner.

The format of these computations is similar to that used in [8]. We use wave speed $c=1$, mesh size $\Delta x=\Delta y=1 / 25$, and mesh ratio $\Delta t / \Delta x=\Delta t / \Delta y=.625$. For all tests the initial condition is

$$
\begin{aligned}
& \text { (a) } u(x, y, 0)= \begin{cases}e^{-30 r^{2}}, & r<.45 \\
0, & r>.45\end{cases} \\
& \text { (b) } u_{t}(x, y, 0)=0,
\end{aligned}
$$

where $r^{2}=(x-.5)^{2}+y^{2}$. The Fourier transform of the Gaussian is a Gaussian centered about wave number zero. A comparison with the graph of the dispersion relation (4.2) shows that the wave motion is composed of Fourier modes associated with all possible directions of propagation. There will therefore be a broad range of angles of incidence present at each boundary used here.

In Tests \#1 and \#2 we compute solutions to the wave equation on the domain

$$
\Omega_{1}=\{(x, y): 0<x<2,-2<y<2\},
$$

with various choices of boundary condition at $x=0$. The solutions are analyzed only on the smaller domain

$$
\Omega_{2}=\{(x, y): 0<x<1,-1.5<y<1.5\} .
$$

(See Figure 8.1(a).) The boundaries of $\Omega_{1}$ are chosen so that reflections from the top, bottom, and right boundaries of $\Omega_{1}$ are not able to reach $\Omega_{2}$ during the time interval on which solutions are computed. The computations thus test reflection properties of the boundary conditions imposed at $x=0$, without interference from any other boundary. The support of the initial data is contained in $\Omega_{2}$.

With the initial condition (8.1) we also compute a solution on the larger domain $\{(x, y):-1<x<2,-2<y<2\}$. The restriction of this solution to $\Omega_{2}$ is the "free-space" solution corresponding to zero reflection. This will be used to compute reflected errors. 


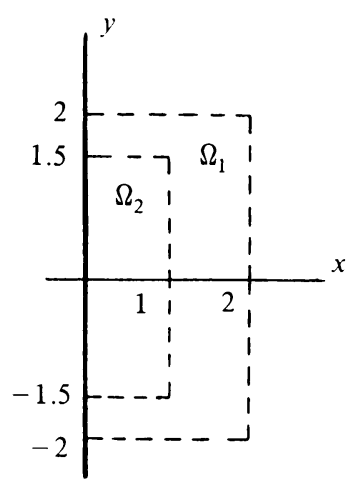

(a) Tests \#1 and \#2

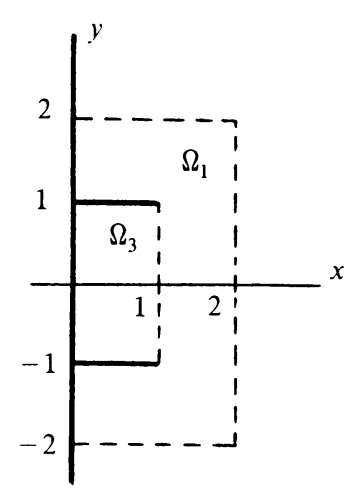

(b) Test \#3

Figure 8.1

Domains used in the computations.

For each computation in Tests $\# 1$ and $\# 2$, we calculate $L^{2}$-norms of the reflection on $\Omega_{2}$ at times $.25, .50, .75, \ldots, 2.00$. These reflections are then expressed as percentages of the $L^{2}$-norm of the initial value (8.1)(a). In the results given in Tables 8.1, 8.2 and 8.3, we give only maximum reflections (from those at the stated times).

Test \#1. Effects of different values of $a$. Here we use the boundary condition

$$
D\left(K, Z^{-1}\right) u_{0, m}^{n+1}=0
$$

with $a=b$. This is the first-order version of (3.3). Solutions are computed using various values of $a$ and with $\alpha=0$ and $\alpha=30$ degrees. ( $\alpha$ is the angle of incidence for which the factor (3.1) is designed to be perfectly absorbing.) The purpose of this set of computations is to illustrate the effects of different choices of $a$, and, in particular, illustrate the stability limit (7.5).

\section{TABLE 8.1}

Test \#1. Percent reflection for first-order boundary condition with $\alpha=0$. For each value of $a$, the amount of reflection given is the maximum observed at times $0.25,0.50, \ldots, 2.0$.

\begin{tabular}{c|rrrrrrrr}
$a$ & -10 & -1 & 0 & 0.1 & 0.2 & 0.25 & 0.3 & 0.4 \\
\hline Reflection & 34.4 & 13.3 & 9.06 & 8.88 & 8.78 & 8.77 & 8.78 & 8.86 \\
$a$ & 0.5 & 0.6 & .65 & .68 & .69 & .70 & .71 & .72 \\
\hline Reflection & 9.03 & 9.28 & 9.43 & 9.54 & 9.57 & 660. & $10^{5}$ & $10^{8}$
\end{tabular}

TABLE 8.2

Test \#1. Percent reflection for first-order boundary condition with $\alpha=30$ degrees.

\begin{tabular}{c|rrrrrrrr}
$a$ & 0 & 0.25 & 0.5 & 0.6 & .67 & .68 & .69 & .70 \\
\hline Reflection & 7.23 & 6.94 & 7.33 & 7.67 & 7.95 & 8.00 & 350. & $10^{5}$
\end{tabular}.


TABLE 8.3

Test \#2. Percent reflection for second-order boundary condition with various combinations of $\alpha_{1}$ and $\alpha_{2}$. The angles are measured in degrees.

$\begin{array}{lcccc} & \alpha_{1}=30 & \alpha_{1}=40 & \alpha_{1}=50 & \alpha_{1}=60 \\ \alpha_{2}=30 & 2.91 & & & \\ \alpha_{2}=40 & 2.71 & 2.57 & & \\ \alpha_{2}=50 & 2.61 & 2.51 & 2.46 & \\ \alpha_{2}=60 & 2.77 & 2.62 & 2.91 & 3.80\end{array}$

The results for $\alpha=0$ are given in Table 8.1. For every value of $a$ except the last three, the maximum reflection from times $.25, .50, \ldots, 2.0$ occurs either at $t=1.25$ or $t=1.50$. For $a=.70, .71$, and .72 the solution displays an obvious instability, and the reflections for these are given at $t=2.0$.

Some results for $\alpha=30$ degrees are given in Table 8.2. The reflections for this case display the same general variation with $a$ that is seen for $\alpha=0$, so here we show mainly the results that illustrate the onset of instability. In general, the reflections for this case are less than those for $\alpha=0$; the reasons for this are included in the discussion of Test \#2.

In Section 7 it was stated that if $a=b, \Delta x=\Delta y$, and $\lambda=.625$, then the stability limit (7.5) says $a<.684$ when $\alpha=0$ and $a<.674$ when $\alpha=30$ degrees. This is consistent with the behavior observed in Tables 8.1 and 8.2.

Test $\# 2$. Effects of different $\alpha_{1}$ and $\alpha_{2}$. Here we use the boundary condition

$$
D_{1}\left(K, Z^{-1}\right) D_{2}\left(K, Z^{-1}\right) u_{0, m}^{n+1}=0
$$

at $x=0$, where $D_{1}\left(K, Z^{-1}\right)$ and $D_{2}\left(K, Z^{-1}\right)$ are discretizations of (3.1) with $\alpha=\alpha_{1}$ and $\alpha=\alpha_{2}$, respectively. Equation (8.3) is a discretization of (1.2) with $p=2$. Solutions are computed using various values of $\alpha_{1}$ and $\alpha_{2}$. In $D_{1}\left(K, Z^{-1}\right)$ and $D_{2}\left(K, Z^{-1}\right)$ we use $a=b=.25$.

If $\alpha_{1}=\alpha_{2}=0$, the maximum percent reflection on $\Omega_{2}$ is 3.48; if $\alpha_{1}=\alpha_{2}=10$ degrees, the reflection is 3.41; if $\alpha_{1}=\alpha_{2}=20$ degrees, the reflection is 3.22. Table 8.3 gives an array of reflections corresponding to various other $\alpha_{1}$ and $\alpha_{2}$. In all of these cases, the maximum reflection from times $0.25,0.50, \ldots, 2.00$ occurs either at $t=1.25, t=1.50$, or $t=1.75$.

In this particular series of computations the minimum reflection is found when $\alpha_{1}$ and $\alpha_{2}$ are each near 50 degrees. As $\alpha_{1}$ and $\alpha_{2}$ are increased from zero, the effect is to spread out the zeros of the reflection coefficient (1.3) and thereby broaden the range of angles of incidence where the boundary condition is highly absorbing. However, as expected, the effectiveness of the boundary condition is reduced if $\alpha_{1}$ and $\alpha_{2}$ are made too large.

Figure 8.2 shows the reflection patterns for the cases $\alpha_{1}=\alpha_{2}=0$ and $\alpha_{1}=\alpha_{2}=$ 50 degrees. In each graph the plotted quantity is the solution computed with boundary minus the free-space solution. The vertical scale is exaggerated in order to make the patterns visible; on the surfaces in Figures 8.2(a) and 8.2(b), the maximum 


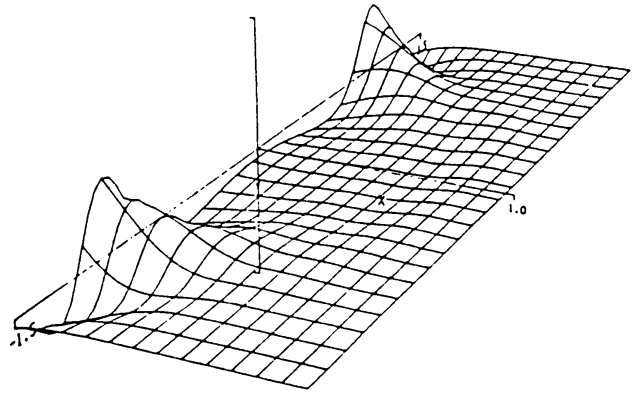

(a) $\alpha_{1}=\alpha_{2}=0$

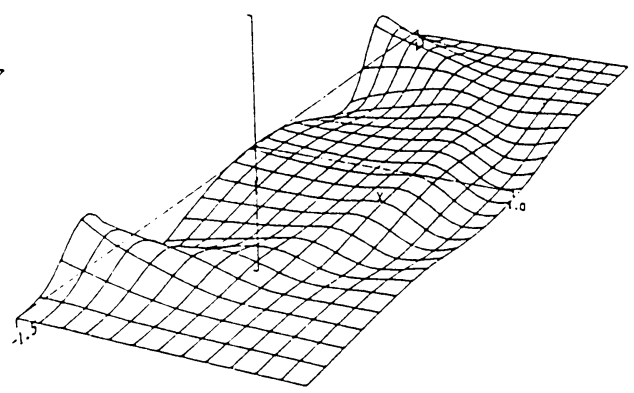

(b) $\alpha_{1}=\alpha_{2}=50$ degrees

FIGURE 8.2

Test \#2. Reflection patterns at time $t=1.25$.

ordinates are 0.022 and 0.014 , respectively. The positive $x$-axis points to the right, and the positive $y$-axis recedes into the background. The graph shows every other grid point in the $x$ direction and every third point in the $y$ direction.

In the above tests, $L^{2}$-norms of reflections were also computed on the interior domain $[1 / 3,1] \times[-1.5,1.5]$. These norms are minimized when $\alpha_{1}$ and $\alpha_{2}$ are each near 45 degrees.

In general, the choice of optimal $\alpha_{1}$ and $\alpha_{2}$ depends on the configuration of the problem. For example, on a short boundary segment, large angles of incidence might not be possible, so $\alpha_{1}$ and $\alpha_{2}$ should be reduced accordingly.

Test \#3. Behavior near a corner. In the analysis given in this paper, the spatial domain is assumed to be a half-space. This can be regarded as a study of a localized problem in a neighborhood of a flat portion of the boundary of a bounded domain. However, this analysis does not consider the effects of the boundary conditions in a neighborhood of a corner. Here we give some empirical evidence which suggests that no difficulties are encountered in this case. It is assumed that an absorbing boundary condition is imposed at each of the intersecting boundary segments. These computations were performed because Engquist and Majda [4] found that instabilities could arise if boundary conditions of their form were matched improperly at the point of intersection.

We compute solutions on the domain $\{(x, y): 0<x<2,-1<y<1\}$ with initial condition (8.1). The solutions are analyzed only on the smaller domain

$$
\Omega_{3}=\{(x, y): 0<x<1,-1<y<1\} .
$$

(See Figure 8.1(b).) The boundary conditions being tested are imposed at $x=0$ and $y= \pm 1$. With the given initial condition we also compute a solution on $\{(x, y)$ : $-1<x<2,-2<y<2\}$; the restriction of this solution to $\Omega_{3}$ is used to calculate reflected errors.

For each boundary condition that is imposed at $x=0$, we also compute a solution on the domain $\Omega_{1}$ defined earlier. The amount of reflection on $\Omega_{3}$ is then calculated. The comparison of the two sets of computations indicates the effects of the boundaries $y= \pm 1$ and the corners $(0,1)$ and $(0,-1)$. 
The following sets of boundary conditions are tested.

(a) Second-order space-time extrapolation at $x=0$ and $y= \pm 1$. This boundary condition uses the square of the operator $S\left(K, Z^{-1}\right)$ defined in (5.1); the condition at the boundary $x=0$ can be written as

$$
u_{0, m}^{n+1}=2 u_{1, m}^{n}-u_{2, m}^{n-1} \text {. }
$$

This method is equivalent to the box scheme and forward Euler discretizations of $(\lambda \partial / \partial t-c \partial / \partial x)^{2} u=0$. Here, $\lambda=.625$, so $\cos ^{-1} \lambda \doteq 51.3$ degrees.

(b) General second-order boundary condition (8.3) at $x=0$, with $\alpha_{1}=\alpha_{2}=30$ degrees and $a=.25$, and second-order space-time extrapolation at $y= \pm 1$.

The discrete boundary conditions used here have one-dimensional stencils, so there is no difficulty with implementing them near the corners. This is different from the case where the boundary conditions involve tangential derivatives. (Cf. [4].) Because of the form of the interior difference scheme (2.2), the corner points are never used in the computation and are excluded from the calculation of reflected errors.

The results of the computations are shown in Figure 8.3. For each computation the percent reflections were calculated for times $.25, .50, \ldots, 2.00$. In each graph the dotted line gives the reflections for the computation with boundary $x=0$, and the solid line gives the reflections with boundaries $x=0$ and $y= \pm 1$. In each case the additional boundaries yield additional reflection, but there is no evidence of any instabilities arising from the effects of the corners.

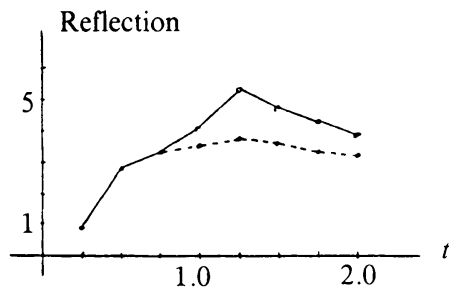

(a)

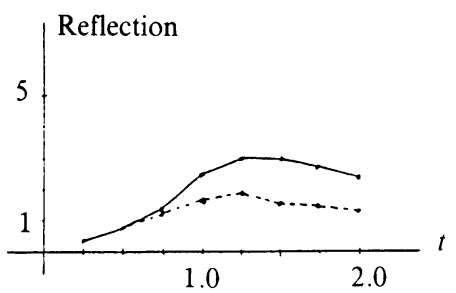

(b)

FIGURE 8.3

Tests \#3. Effects of a corner. In each of cases (a) and (b), the dotted line gives the percent reflection without a corner, and the solid line gives reflections with a corner.

Note Added in Proof. It has recently come to the author's attention that R. G. Keys has independently derived analytical boundary conditions of the form (1.2). See Geophysics, vol. 50, no. 6, June 1985, pp. 892-902.

Department of Mathematics

Oregon State University

Corvallis, Oregon 97331

1. A. Bamberger, B. Engquist, L. Halpern \& P. Joly, Construction et Analyse d'Approximations Paraxiales en Milieu Hétérogène. I and II, Internal Reports 114 and 128, Centre de Mathématiques Appliquées, Ecole Polytechnique, 1984-1985.

2. A. Bayliss \& E. TURKel, "Radiation boundary conditions for wave-like equations," Comm. Pure Appl. Math., v. 33, 1980, pp. 707-725. 
3. B. EngQuist \& A. MAJDA, "Absorbing boundary conditions for the numerical simulation of waves," Math. Comp., v. 31, 1977, pp. 629-651.

4. B. ENGQUIST \& A. MAJDA, "Radiation boundary conditions for acoustic and elastic wave calculations," Comm. Pure Appl. Math., v. 32, 1979, pp. 313-357.

5. B. GustafsSon, H.-O. Kreiss \& A. Sundström, "Stability theory of difference approximations for mixed initial boundary value problems. II," Math. Comp., v. 26, 1972, pp. 649-686.

6. L. Halpern \& L. N. Trefethen, "Wide-angle one-way wave equations," J. Acoust. Soc. Amer. (To appear.)

7. R. L. Higdon, "Initial-boundary value problems for linear hyperbolic systems," SIAM Rev., v. 28, 1986, pp. 177-217.

8. R. L. HigDON, "Absorbing boundary conditions for difference approximations to the multi-dimensional wave equation," Math. Comp., v. 47, 1986, pp. 437-459.

9. E. L. Lindman, “"Free-space” boundary conditions for the time dependent wave equation," $J$. Comput. Phys., v. 18, 1975, pp. 66-78.

10. L. N. Trefethen, "Instability of difference models for hyperbolic initial boundary value problems," Comm. Pure Appl. Math., v. 37, 1984, pp. 329-367.

11. L. N. TREFEThen \& L. HALPERN, "Well-posedness of one-way wave equations and absorbing boundary conditions," Math. Comp., v. 47, 1986, pp. 421-435.

12. G.-Q. Zhang, High Order Approximation of One-Way Wave Equations, Technical report, Computing Center, Academia Sinica, Beijing, China, 1984. 